\title{
The calibration of interferometric visibilities obtained with single-mode optical interferometers
}

\section{Computation of error bars and correlations ${ }^{\star}$}

\begin{abstract}
G. Perrin ${ }^{\star}$
LESIA, FRE 2461, Observatoire de Paris, section de Meudon, 5 place Jules Janssen, 92190 Meudon, France

Received 4 September 2002 / Accepted 19 December 2002

Abstract. I present in this paper a method to calibrate data obtained from optical and infrared interferometers. I show that correlated noises and errors need to be taken into account for a very good estimate of individual error bars but also when model fitting the data to derive meaningful model parameters whose accuracies are not overestimated. It is also shown that under conditions of high correlated noise, faint structures of the source can be detected. This point is important to define strategies of calibration for difficult programs such as exoplanet detection. The limits of validity of the assumptions on the noise statistics are discussed.
\end{abstract}

Key words. techniques: interferometric - methods: data reduction

\section{Introduction}

With optical-infrared interferometry becoming more mature, the quality of visibility measurements have become an issue. Single-mode interferometers (see Sect. 2.3) allow one to eliminate non-stationary effects by filtering out the spatial modes of turbulence. The response of interferometers is therefore very stable and the issue of estimating the accuracies of non-biased data is raised. The final visibility estimate is a complex quantity as it is a non-linear mix of noisy measurements and of parameter estimates with their own uncertainties. Estimating the stability of the instrument, a crucial point for calibration, and the final error on visibilities is therefore non-trivial and must be considered with caution. Moreover, data analysis mainly consists of model fitting the final visibilities and the matter of their potential correlations becomes important, especially if some very faint structures are looked for, as is the case in extra-solar planet detection.

In this Paper I propose a method to meet these challenges. The method has been tested and elaborated along with the FLUOR interferometer, the first single-mode interferometer. This method was first published in Perrin (1996) and used in Perrin et al. (1998). It is updated and improved in this paper by accounting for correlations.

\section{Principles of data reduction and calibration}

In this section the general scheme of data reduction is reviewed to introduce the vocabulary and notations. Two main steps are

\footnotetext{
* Appendix A is only available in electronic form at http://www .edpsciences.org

$\star \star$ e-mail: guy.perrin@obspm.fr
}

to be considered. In the first one (Sect. 2.1), the fringe processing, fringe contrasts are derived from raw signals. Because of contrast losses, fringe contrasts are calibrated in a second step (Sect. 2.2) to provide the visibilities directly linked to the spatial intensity distribution of the source.

\subsection{Fringe contrasts estimates}

In the following we will distinguish between the fringe contrast obtained on a source and the visibility of this source. The fringe contrast measured from a single exposure or scan is called the coherence factor and is noted $\mu$ whereas the visibility is noted $V$.

Whatever the beamcombining technique, $\mu$ being the modulus of a complex number, unbiased estimators are only obtained for squared quantities from wich biases due to additive noise can be subtracted. In the future, phase referencing techniques may allow one to directly measure complex visibilities (real and imaginary parts) but this is not the case yet and I will only consider measurements of fringe contrast moduli. The result of the processing of a series of scans on a single source is a series of realizations of the $\mu^{2}$ estimator or is an average value of the realizations with a $1 \sigma$ error bar if their statistical distribution can be trusted to be Gaussian.

\subsection{Necessity for a calibration}

The average $\mu^{2}$ is not directly an estimator of the squared modulus of the visibility of the source because some physical phenomena degrade the coherence factor. Among these phenomena, polarization mismatches between the interferometric beams are the most common. Without perfect beam cleaning by 

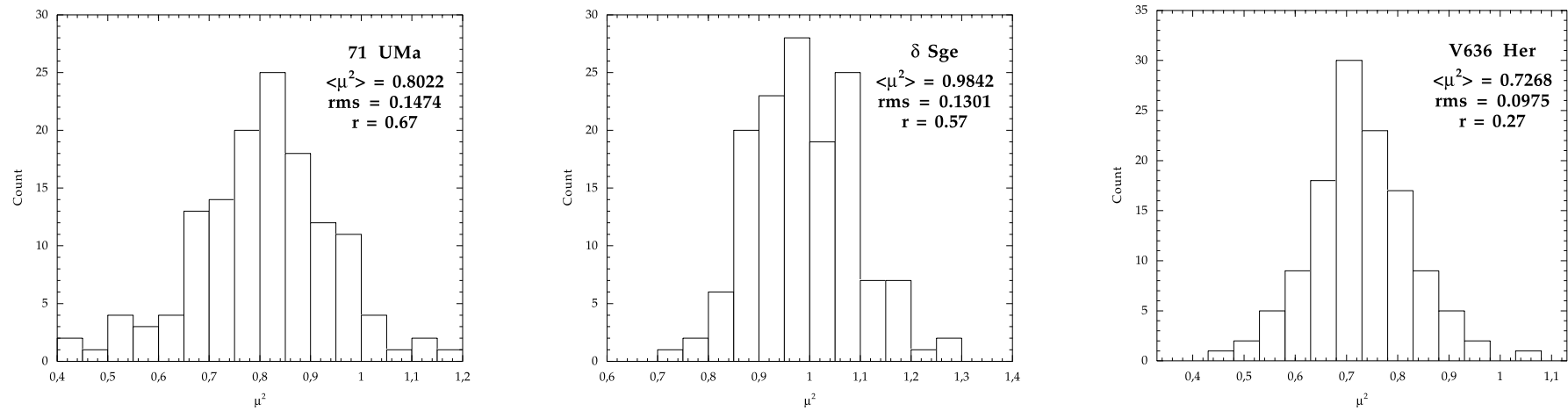

Fig. 1. Examples of squared coherence factor histograms obtained with FLUOR in one of the interferometric channels. About 100 interferograms have been recorded for each object. The mean and rms of individual measurements are given for this channel. The correlation factor $r$ measures the noise correlation between the two interferometric channels. The amount of atmospheric piston is decreasing from the left to the right.

a fiber, atmospheric turbulence also degrades the fringe contrast. It is necessary to estimate the loss of coherence on a calibrator source for which the visibility is known. A transfer function $T$ is obtained by computing the ratio of the measured coherence factor $\mu_{c}$ to the expected visibility $V_{\exp }$. With squared quantities this yields:

$T^{2}=\frac{\mu_{c}^{2}}{V_{\exp }^{2}}$.

If the instrument is stable enough then the estimate of $T^{2}$ obtained on the calibrator can be used to derive an estimated visibility for the source from the measured coherence factor:

$V^{2}=\frac{\mu^{2}}{T^{2}}=\operatorname{coT}^{2} \mu^{2}$.

where I call $c o T^{2}$ the squared co-transfer function. Its use will be detailed in Sect. 4.

\subsection{Assumptions in the case of a single-mode interferometer}

The calibration process may fail if the assumption that the transfer function is stable is wrong. This usually happens if non-stationary processes like atmospheric phase turbulence play a role in the fringe formation process. In a perfect singlemode interferometer in which single-mode fibers are used to filter the phase aberrations produced by atmospheric turbulence (except for the piston mode) these non-stationary effects are eliminated. In order to avoid instabilities due to the piston mode, the fringes are scanned at a frequency far above the characteristic frequency of piston. In interferometers where the piston is stabilized by a fringe tracking servo loop, this issue is solved. The remaining main sources of variation of the transfer function are basically temperature drifts and differential polarisation effects due to the change of beam inclination on the first mirrors with changing positions of the sources in the sky. In both cases the transfer function drifts are very slow and a good estimate of the transfer function can be obtained by interpolating two estimates bracketing the source to be calibrated. This has been demonstrated with the FLUOR beamcombiner, as will be shown is Sect. 7.2.
In the following we will therefore consider that the efficiency of the interferometer is continuously assessed by observing calibrators before and after science sources. We will not consider the case where the transfer function is derived by averaging individual transfer functions on a large temporal scale, as this is not required for a single-mode interferometer. This technique does not allow one to assess the quality of the calibration in detail. Nevertheless, should the transfer function estimates be statistically compatible with a constant transfer function, it would be legitimate to use this value to calibrate a whole night. The method to evaluate correlations should be considered and accordingly adapted.

\section{Estimating fringe contrasts}

This section focuses on estimating the statistical properties of fringe contrasts. I will not describe the method to compute coherence factors from single exposures and I will refer the reader to appropriate articles in the next paragraphs.

\subsection{Single-channel spatial modulation interferometer}

In a multiaxial interferometer, distant parallel beams feed a focusing optic. The beams are recombined in the focal plane where they overlap at the focus locus. The modulation is spatial as the fringe phase varies across the diffraction pattern. A method to derive fringe contrasts has been published by Mourard et al. (1994) in the case of GI2T. The method has been adapted to AMBER which is a single-mode multiaxial interferometer (Chelli et al. 2000).

Thanks to the filtering of the non-stationary modes of turbulence, the statistics of $\mu^{2}$ can be well approximated by a Gaussian distribution. This will be demonstrated in the case of the data obtained with FLUOR in Sect. 7.1. The estimate of the squared coherence factor is therefore the mean of the distribution of the realizations denoted $\overline{\mu^{2}}$. An unbiased estimate of the variance of individual measurements is:

$S^{2}=\frac{1}{N-1} \sum_{n=0}^{N-1}\left(\mu_{n}^{2}-\overline{\mu^{2}}\right)$. 

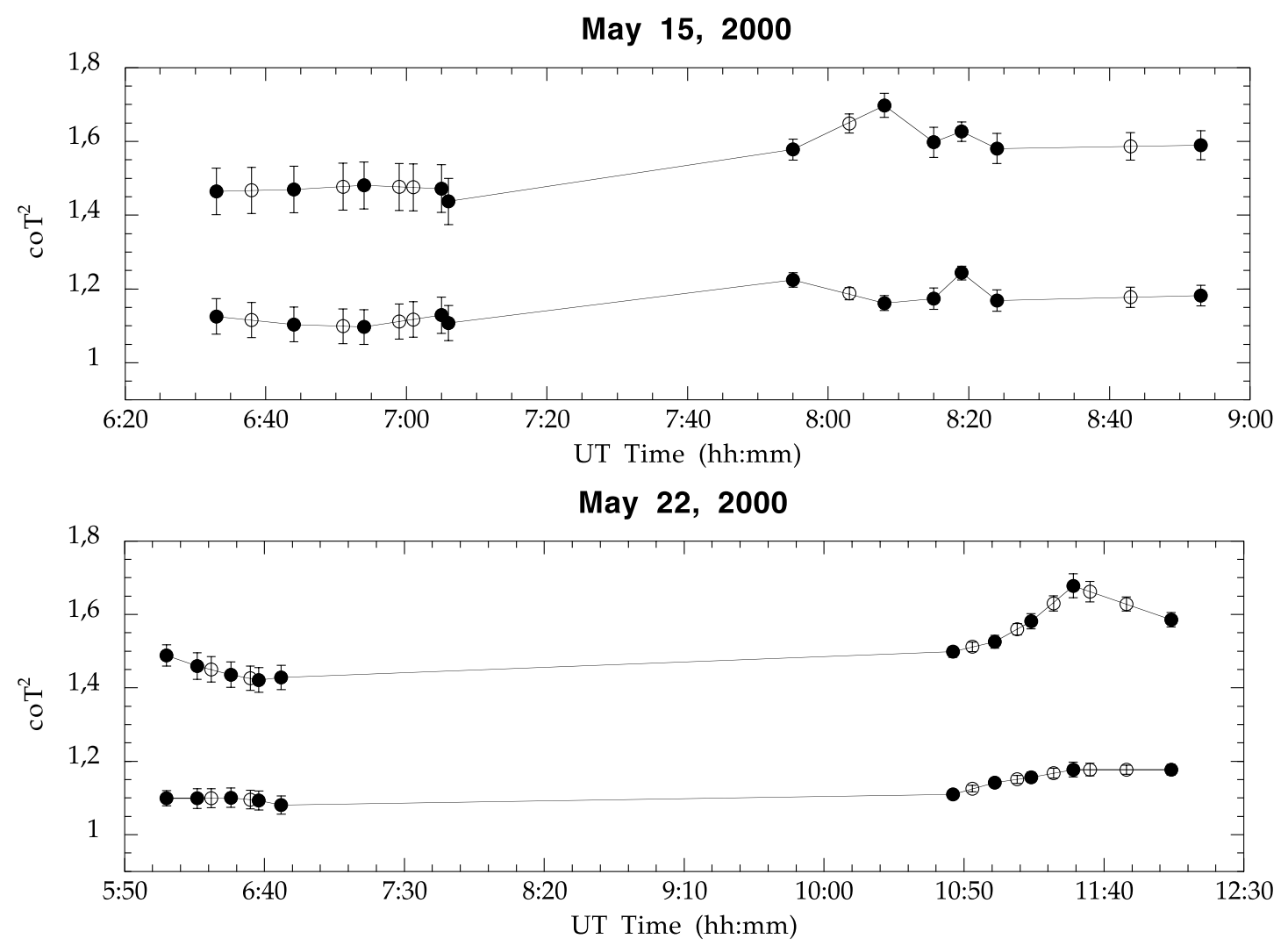

Fig. 2. Examples of squared co-transfer functions measured with FLUOR. The two curves for each night correspond to the two interferometric channels of the coaxial interferometer. The full circles are the squared co-transfer functions measured on calibrators whereas the open circles are the values interpolated at the time when the science targets were observed. $1 \sigma$ error bars are displayed.

The estimate of the variance of the coherence factor estimator $\overline{\mu^{2}}$ is then:

$\operatorname{Var}\left(\overline{\mu^{2}}\right)=\frac{S^{2}}{N}$

\subsection{Two-channel temporal modulation interferometer}

In a coaxial interferometer, beams are superimposed in position and in direction. This can be realized with a beamsplitter or with a fiber coupler. A relative phase between the beams is introduced by setting an optical path difference. This is achieved with a moving mirror in one of the two beams, hence the temporal modulation of the phase. A method to compute fringe contrasts for this type of interferometer is described in Coudé du Foresto et al. (1997). A more recent method based on wavelets analysis has been proposed by Segransan et al. (1999). The method to obtain an estimate of the coherence factor without the photon noise bias is explained in Perrin (2003). A prototype instrument for this kind of interferometer is the FLUOR beamcombiner.

The difference with the previous interferometer of Sect. 3.1 is that it produces two interferometric beams and therefore two sets of coherence factors estimates. The statistics of each set can be well approximated by a Gaussian statistics as will be shown in Sect. 7. The photon noises of the two interferometric signals are uncorrelated. The read-out noises are generally considered uncorrelated but some correlation may occur as different pixels share the same read-out electronics. In addition the two beams suffer from the same turbulence effects (residual piston and photometric beam fluctuations) which generate some noise in the measurements. Part of the noise is therefore common to the two signals and the coherence factors estimates are correlated. The correlation factor $r$ is directly estimated from the $\mu^{2}$ distributions:

$$
r=\frac{\left\langle\mu_{1}^{2}-\overline{\mu_{1}^{2}}\right\rangle\left\langle\mu_{2}^{2}-\overline{\mu_{2}^{2}}\right\rangle}{\sqrt{\operatorname{Var}\left(\mu_{1}^{2}\right) \operatorname{Var}\left(\mu_{2}^{2}\right)}}
$$

where the subscripts describe the two interferometric channels.

\section{Estimating the transfer function}

It is assumed that the transfer function is a slowly varying function which is rapidly sampled. This property will be illustrated with real data in Sect. 7. It is then legitimate to linearly interpolate the squared transfer function at the time when the science source was observed. Because the variances of products of random variables are more easily calculated than those of ratios, the reciprocal of the squared transfer function, the squared cotransfer function, is interpolated instead of the squared transfer function. The use of one or the other is equivalent. In order to be general, two interferometric outputs are always considered. The particular case of the multiaxial interferometer will 


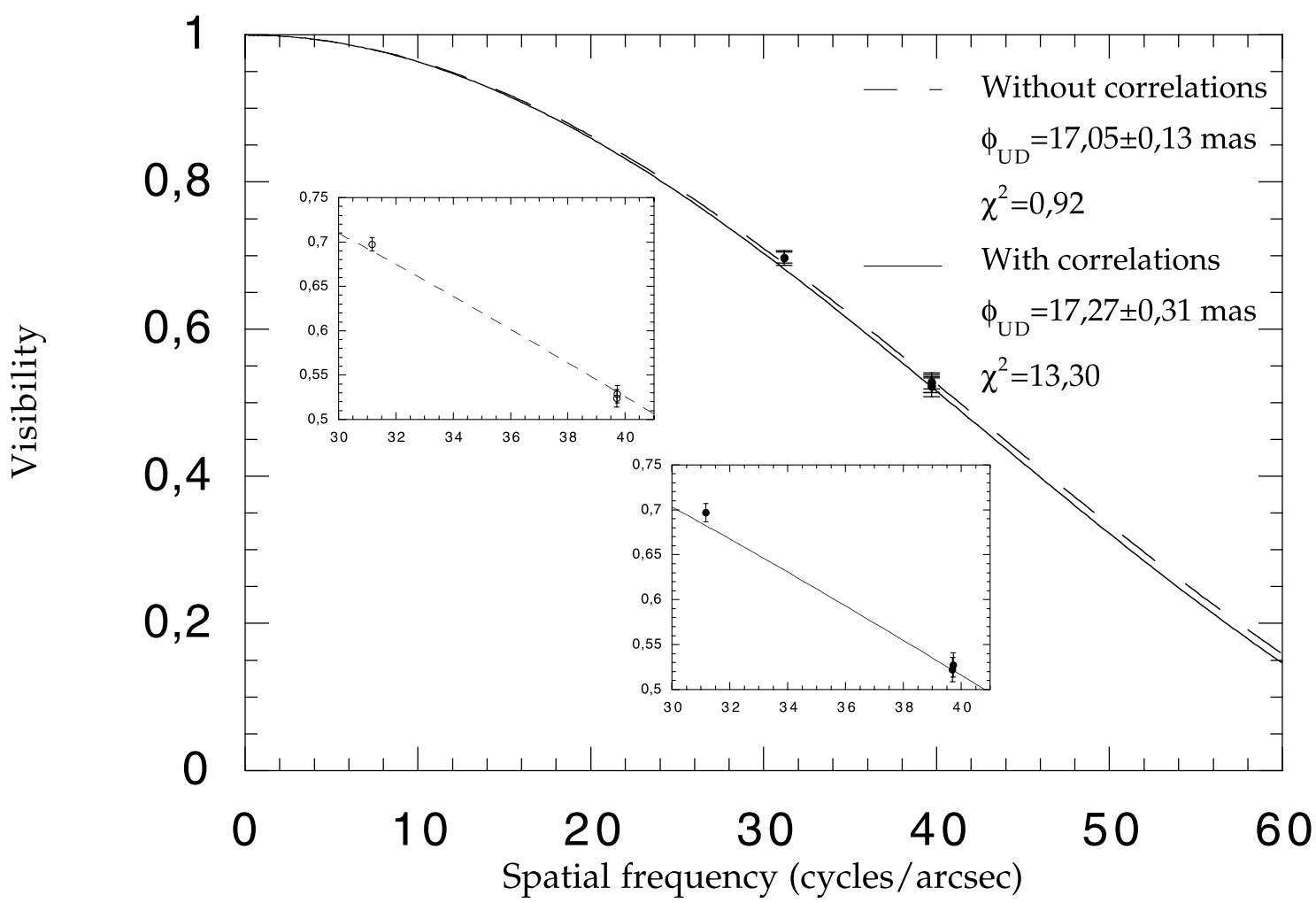

Fig. 3. Example of visibility fit. The source is SW Vir and the model is a uniform disk. Errors are $1 \sigma$ errors. Open circles and dashed line are the visibilities and model fit computed without taking correlations into account. Full circles and continuous line are the equivalent with the method described in this paper. The two cases are separately presented in the little windows.

be considered in discussions. The expression of the interpolated co-transfer functions in the two outputs of the instrument is:

$\left\{\begin{array}{l}c o T_{1}^{2}=x \frac{V^{a 2}}{\overline{\mu_{1}^{a 2}}}+(1-x) \frac{V^{b^{2}}}{\overline{\mu_{1}^{b^{2}}}} \\ \operatorname{coT}_{2}^{2}=x \frac{V^{a 2}}{\overline{\mu_{2}^{a 2}}}+(1-x) \frac{V^{b^{2}}}{\overline{\mu_{2}^{b^{2}}}}\end{array}\right.$

$V^{a}$ and $V^{b}$ are the expected visibilities of calibrators $\mathrm{A}$ and $\mathrm{B}$. Coefficient $x$ is the relative time distance between the observation of the science target and the observation of calibrator A. The expected visibilities are supposed to be Gaussian random variables. Dropping the channel indices, the variances of the squared co-transfer functions are equal to:

$\operatorname{Var}\left(c o T^{2}\right)=x^{2} \operatorname{Var}\left(c o T^{a 2}\right)+(1-x)^{2} \operatorname{Var}\left(c o T^{b^{2}}\right)$

when the two calibrators are different. When the two calibrators are the same then the variance is equal to:

$$
\begin{aligned}
& \operatorname{Var}\left(c o T^{2}\right)=x^{2} \operatorname{Var}\left(c o T^{a 2}\right)+(1-x)^{2} \operatorname{Var}\left(c o T^{b^{2}}\right) \\
& +2 \frac{x(1-x)}{\overline{\mu^{a 2}} \overline{\mu^{b^{2}}}} \operatorname{Var}\left(V^{a 2}\right) \text {. }
\end{aligned}
$$

The squared co-transfer functions estimated on the calibrators are ratios of Gaussian estimators. These new random distributions are not Gaussian. They are Cauchy distributions (the density probability of which is a Lorentzian) with no mean and no variance. By analogy with the standard deviation of a Gaussian law, an estimate of the uncertainty can be derived from the width of the confidence interval. The upper and lower limits of the confidence interval at $68.3 \%$ for a ratio of two Gaussian random variables $\alpha$ and $\beta$ are given by (Pelat 1992):

$L_{ \pm}=\frac{\alpha \pm \frac{\beta / \sigma_{\beta}}{\left(\left(\alpha / \sigma_{\alpha}\right)^{2}+\left(\beta / \sigma_{\beta}\right)^{2}-1\right)^{1 / 2}} \sigma_{\alpha}}{\beta \mp \frac{\alpha / \sigma_{\alpha}}{\left(\left(\alpha / \sigma_{\alpha}\right)^{2}+\left(\beta / \sigma_{\beta}\right)^{2}-1\right)^{1 / 2}} \sigma_{\beta}}$

This interval is not symmetric. I choose as error bar the larger distance of the mean to the limits, thus slightly overestimating the error. The probability that the true value is in this interval around the mean is therefore larger than $68.3 \%$. In the following I will consider that ratios of Gaussian variables are Gaussian variables. This is not rigourously true but this allows one to derive expressions otherwise difficult to handle. The Gaussian and Lorentzian functions are both bell curves, the wings of the latter being more extended than that of the Gaussian. The approximation amounts to giving more weight to the center of the lorentzian. The other possibility would be to apply Monte-Carlo or bootstrap methods to all error bar computations, which would make data reduction a very long process for a very limited gain. Nevertheless, this method will have to be applied once to compute the correlation between the visibilities of a two-channel coaxial interferometer (Sect. 5). Consistency of error bars will be addressed in Sect. 7. This final consistency is the justification of the approximations performed. 
X Her

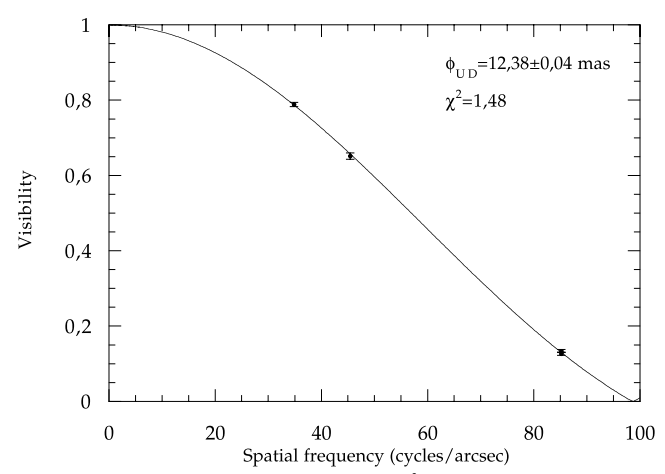

BK Vir

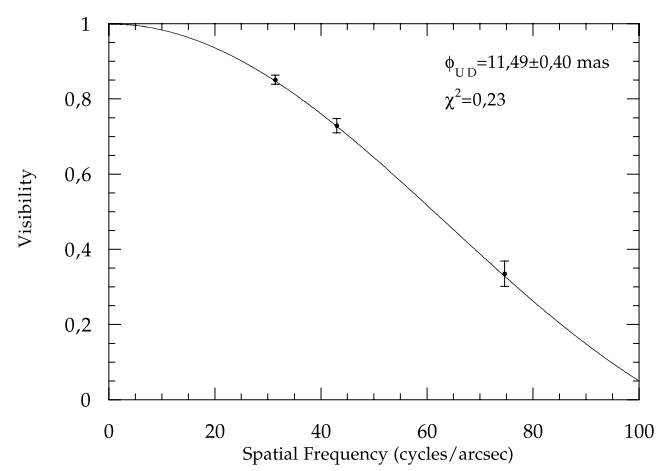

G Her

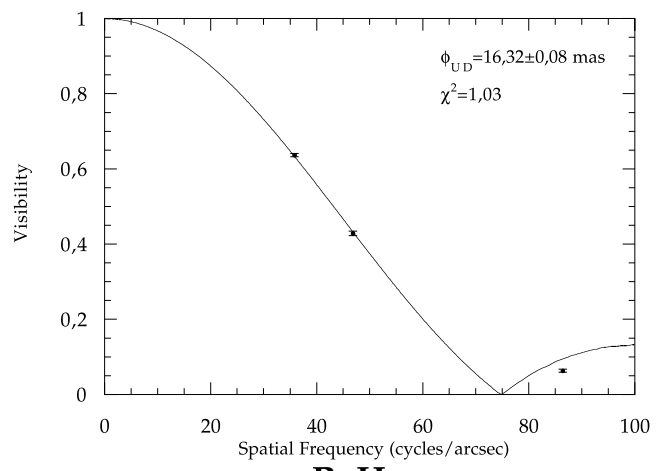

R Hya

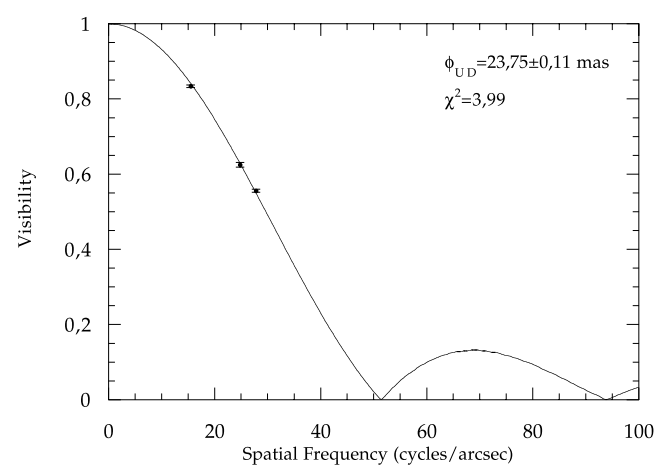

Fig. 4. Examples of fits of visibility data with a uniform disk model. All correlations are taken into account. Errors are $1 \sigma$ errors. The visibility point around 85 cycles/arcsec in the G Her panel is not taken into account in the fit.

\section{Estimating visibilities}

\subsection{Mean and variance of channel visibilities}

The single-channel squared visibility in the case of a multiaxial interferometer or the two-channel squared visibilities of a coaxial interferometer are simply the product of the science target squared coherence factors and squared co-transfer functions of Eq. (6) yielding:

$\left\{\begin{array}{l}V_{1}^{2}=x \frac{\overline{\mu_{1}^{2}}}{\frac{\mu_{1}^{a 2}}{\mu_{2}^{2}}} V^{a 2}+(1-x) \frac{\overline{\mu_{1}^{2}}}{\frac{\frac{\mu_{1}^{b^{2}}}{\mu^{2}}}{\mu_{2}^{2}}} V^{b^{2}} \\ V_{2}^{2}=x \frac{\frac{\mu_{2}^{a 2}}{\mu_{2}^{b_{2}^{2}}}}{{ }^{b_{2}}} V^{b^{2}} .(1-\end{array}\right.$

The variances of the two-channel squared visibilities are calculated with the variances of the squared co-transfer functions and of the squared coherence factors with the following formula:

$$
\begin{aligned}
\operatorname{Var}(A B)=\quad & \operatorname{Var}(A) \operatorname{Var}(B)+\bar{A}^{2} \operatorname{Var}(B) \\
& +\bar{B}^{2} \operatorname{Var}(A)
\end{aligned}
$$

We therefore have:

$$
\begin{aligned}
\operatorname{Var}\left(V_{1}^{2}\right)= & \operatorname{Var}\left(\overline{\mu_{1}^{2}}\right) \operatorname{Var}\left(c o T_{1}^{2}\right)+\overline{\mu_{1}^{2}} \operatorname{Var}\left(c o T_{1}^{2}\right) \\
& +c o T_{1}^{2} \operatorname{Var}\left(\overline{\mu_{1}^{2}}\right) \\
\operatorname{Var}\left(V_{2}^{2}\right)= & \operatorname{Var}\left(\overline{\mu_{2}^{2}}\right) \operatorname{Var}\left(c o T_{2}^{2}\right)+\overline{\mu_{2}^{2}} \operatorname{Var}\left(c o T_{2}^{2}\right) \\
& +c o T_{2}^{2} \operatorname{Var}\left(\overline{\mu_{2}^{2}}\right)
\end{aligned}
$$

\subsection{Correlation between two channel visibilities}

The above equations define the uncertainties on the channel estimates of the visibilities. In the case of a multiaxial interferometer, this is the final estimate of the visibility. In the case of coaxial interferometers, the two estimates of the visibility need to be averaged at this stage. For this, it is necessary to assess the correlation factor between the two estimates. By definition, the correlation factor is equal to:

$\rho_{12}=\frac{\left\langle\left(V_{1}^{2}-\overline{V_{1}^{2}}\right)\left(V_{2}^{2}-\overline{V_{2}^{2}}\right)\right\rangle}{\sqrt{\operatorname{Var}\left(V_{1}^{2}\right) \operatorname{Var}\left(V_{2}^{2}\right)}}$.

This quantity is defined by sums, ratios and products of ten random variables. The correlation factor has to be computed with a Monte-Carlo method by simulating each random variable from its mean and variance (assuming it has Gaussian statistics) and by correlating the series of $V_{1}^{2}$ and $V_{2}^{2}$. In the special case when the correlations between measured quantities can be neglected because the correlated noise level is far below that of the uncorrelated noise, it is to be noticed that there is still a correlation due to the common estimated values of the calibrators expected visibilities in the two interferometric channels. This can be illustrated by the equation below when the first and second calibrators are different:

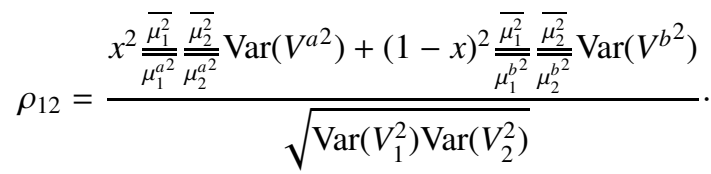


When the two calibrators are the same the correlation factor has the particular expression (for sake of simplicity the two estimates of the visibility at slighlty different baselines are supposed to be the same):

$$
\rho_{12}=\frac{\left[x \frac{\overline{\mu_{1}^{2}}}{\overline{\mu_{1}^{a}}}+(1-x) \frac{\overline{\mu_{1}^{2}}}{\frac{\mu_{1}^{b^{2}}}{2}}\right]\left[x \frac{\overline{\mu_{2}^{2}}}{\mu_{2}^{a 2}}+(1-x) \frac{\overline{\mu_{2}^{2}}}{\overline{\mu_{2}^{b^{2}}}}\right] \operatorname{Var}\left(V^{a 2}\right)}{\sqrt{\operatorname{Var}\left(V_{1}^{2}\right) \operatorname{Var}\left(V_{2}^{2}\right)}} .
$$

It is now easy to see from Eq. (10) that if the noise on the measurements is negligible with respect to the uncertainties on the expected visibilities of the calibrators then the correlation tends towards 1 . In this case, the second interferometric channel brings no extra information except a consistency check and the precision on the visibility of the science target is directly proportional to the precision on the expected visibilities of the calibrators.

\subsection{Comments on visibility variances}

It is also interesting to analyze the propagation of noises in the visibility estimates. For example, if the noise on the measurements is negligible, it is possible to evaluate the amount of variance due to the uncertainty on the calibrators visibilities (or diameters). Dropping channel indices I obtain:

$$
\begin{aligned}
\left.\operatorname{Var}\left(V^{2}\right)\right|_{\mu, \mu^{a}, \mu^{b}}= & x^{2}\left[\frac{\overline{\overline{\mu^{2}}}}{\overline{\mu^{a 2}}}\right]^{2} \operatorname{Var}\left(V^{a 2}\right) \\
& +(1-x)^{2}\left[\frac{\overline{\overline{\mu^{2}}}}{\overline{\mu^{b^{2}}}}\right]^{2} \operatorname{Var}\left(V^{b^{2}}\right) .
\end{aligned}
$$

If the uncertainties on the calibrators' squared visibilities are equal to $1 \%$ and if the coherence factors are all equal to 1 and observations are equally spaced in time then the uncertainty on the measured squared visibility is equal to $0.7 \%$. This equation also shows that the smaller the visibility of the calibrator, the more amplified the noise on its expected visibility is. Symmetrically, the smaller the visibility of the science target, the smaller the contribution to the noise of the calibrators' expected visibilities.

\subsection{Final estimate of the visibility in a two-channel interferometer}

The final squared visibility $V^{2}$ is estimated from the two squared visibilities obtained from each output of the interferometer $V_{1}^{2}$ and $V_{2}^{2}$ and their respective variances (or equivalently uncertainties $\sigma_{1}$ and $\sigma_{2}$ ). I define the final estimate $V^{2}$ as being the least squares fit estimator of the squared visibility as this is an optimal estimator for Gaussian random variables. In this fit, the model is linear and has only one parameter: $V^{2}$. Let us call $C$ the covariance matrix of $V_{1}^{2}$ and $V_{2}^{2}$ :

$C=\left[\begin{array}{cc}\sigma_{1}^{2} & \rho_{12} \sigma_{1} \sigma_{2} \\ \rho_{12} \sigma_{1} \sigma_{2} & \sigma_{2}^{2}\end{array}\right]$.
The quantity to minimize in the least squares fit can then be written:

$S\left(V^{2}\right)=\left[\begin{array}{l}V_{1}^{2}-V^{2} \\ V_{2}^{2}-V^{2}\end{array}\right]^{t} C^{-1}\left[\begin{array}{c}V_{1}^{2}-V^{2} \\ V_{2}^{2}-V^{2}\end{array}\right]=Y^{t} C^{-1} Y$.

It can be shown that the minimum is reached for:

$V^{2}=\left(X^{t} C^{-1} X\right)^{-1} X^{t} C^{-1}\left[\begin{array}{l}V_{1}^{2} \\ V_{2}^{2}\end{array}\right]$,

with

$X=\left[\begin{array}{l}1 \\ 1\end{array}\right]$

the uncertainty on $V^{2}$ being:

$\sigma_{V^{2}}^{2}=\left(X^{t} C^{-1} X\right)^{-1} X^{t} C^{-1}$.

The above equations yield the final visibility estimate:

$V^{2}=\frac{V_{1}^{2}\left(\sigma_{2}^{2}-\rho_{12} \sigma_{1} \sigma_{2}\right)+V_{1}^{2}\left(\sigma_{1}^{2}-\rho_{12} \sigma_{1} \sigma_{2}\right)}{\sigma_{1}^{2} \sigma_{2}^{2}-2 \rho_{12} \sigma_{1} \sigma_{2}}$

and the associated error:

$\sigma_{V^{2}}^{2}=\frac{\left(1-\rho_{12}^{2}\right) \sigma_{1}^{2} \sigma_{2}^{2}}{\sigma_{1}^{2}+\sigma_{2}^{2}-2 \rho_{12} \sigma_{1} \sigma_{2}}$.

If $\rho_{12}=1$ then the two single-output squared visibilities $V_{1}^{2}$ and $V_{2}^{2}$ are fully correlated and the above expression does not apply. In this case $\mathrm{V}$ is equal to one of the two single-output visibilities with its associated error bar.

The quality of the fit is expressed by the $\chi^{2}$ :

$\chi^{2}=\frac{\left(V_{1}^{2}-V_{2}^{2}\right)^{2}}{\sigma_{1}^{2}+\sigma_{2}^{2}-2 \rho_{12} \sigma_{1} \sigma_{2}}$.

This parameter is important because it allows us to check the consistency of the instrument and of the method to measure the visibilities and the error bars. If all assumptions are correct then the $\chi^{2}$ should be equal to 1 on average. In the FLUOR software we use this number as a data quality parameter. Data with $\chi^{2}$ greater than 3 should be examined in detail and rejected for science programs requiring a very good quality of calibration as the probability to get a value larger than 3 is only of $8.33 \%$.

\subsection{Correlations of multiple baseline interferometer simultaneous visibilities}

Coherence factors recorded simultaneously on different baselines with telescopes in common may also be correlated. This correlation should be taken into account and saved with the reduced data in the form of a correlation matrix. The correlations may be as high as the correlations between the two channels of a coaxial interferometer as all calibrators are common to all baselines. The method used in Sect. 5.2 should be applied. A correlation matrix for the $\overline{\mu^{2}}$ should be computed first. The final correlation factors for the final visibility estimates are then computed with a Monte-Carlo method. 


\section{Correlations between non-simultaneous visibilities}

Visibilities obtained on different baselines or on different days are usually considered independent. In the last paragraph, we focused on the possible correlations of visibilities recorded simultaneously on baselines with telescopes in common. In this section, we will consider the correlation due to common uncertainties in the calibration process for independent baselines or for visibilities measured at different times. The calibration of the transfer function may have required us to use the same calibrators hence the same diameter estimates. The errors on the visibilities are therefore not independent. It is the purpose of this paragraph to establish a method to compute this correlation and, more important, to be able to trace it to compute it a posteriori long after taking the data at the telescopes.

Let $S_{1}$ and $S_{2}$ be two spatial frequencies at which squared visibilities $V^{2}\left(S_{1}\right)$ and $V^{2}\left(S_{2}\right)$ have been measured. The visibility estimates of Eqs. (10) and (22) can take the form:

$\left\{\begin{array}{l}V^{2}\left(S_{1}\right)=\alpha_{1} V^{a 2}\left(S_{1}\right)+\beta_{1} V^{b^{2}}\left(S_{1}\right) \\ V^{2}\left(S_{2}\right)=\alpha_{2} V^{c 2}\left(S_{2}\right)+\beta_{2} V^{d^{2}}\left(S_{2}\right)\end{array}\right.$

where the calibrators are A, B, C and D. To save room, the calibrator visibilities are replaced by the capital letters. It can be shown that the correlation factor between the two squared visibilities is:

$$
\begin{aligned}
\rho\left(V^{2}\left(S_{1}\right), V^{2}\left(S_{2}\right)\right)= & \frac{\alpha_{1} \alpha_{2} \sqrt{\operatorname{Var}(A) \operatorname{Var}(B)} \rho(A, C)}{\sqrt{\operatorname{Var}\left(V^{2}\left(S_{1}\right)\right) \operatorname{Var}\left(V^{2}\left(S_{2}\right)\right)}} \\
& +\frac{\alpha_{1} \beta_{2} \sqrt{\operatorname{Var}(A) \operatorname{Var}(D)} \rho(A, D)}{\sqrt{\operatorname{Var}\left(V^{2}\left(S_{1}\right)\right) \operatorname{Var}\left(V^{2}\left(S_{2}\right)\right)}} \\
& +\frac{\beta_{1} \alpha_{2} \sqrt{\operatorname{Var}(B) \operatorname{Var}(C)} \rho(B, C)}{\sqrt{\operatorname{Var}\left(V^{2}\left(S_{1}\right) \operatorname{Var}\left(V^{2}\left(S_{2}\right)\right)\right.}} \\
& +\frac{\beta_{1} \beta_{2} \sqrt{\operatorname{Var}(B) \operatorname{Var}(D)} \rho(B, D)}{\sqrt{\operatorname{Var}\left(V^{2}\left(S_{1}\right)\right) \operatorname{Var}\left(V^{2}\left(S_{2}\right)\right)}} .
\end{aligned}
$$

When all calibrators are different, all correlations are zero. The correlation is maximum when a single calibrator has systematically been used. Measurement noise is present at the denominator only and the correlation is of course all the larger as the measurement noise is smaller.

The correlation between two expected squared visibilities at two different baselines is not easy to evaluate analytically. Besides, it may depend upon the model of the calibrator. A computation can be performed which shows that the correlation is indeed equal to 1 with an excellent accuracy as long as no baseline is equal to 0 . This can also be shown by expanding the visibility function. Thus, the expected visibilities derived from a uniform disk model of a same calibrator at two different baselines are fully correlated to the first order. This holds as long as the second derivative of the model is small (which in the case of the uniform disk model is true except close to the zeros of the model) and as a condition, none of the baselines is very close to zero. In practice, the error on the diameter being usually small (less than 5\%), the first order approximation is valid and the two expected squared visibilities can therefore be considered fully correlated. This is true down to very short baselines as for example for a diameter of $10 \pm 0.5$ mas the correlation starts to decrease for a baseline below $5 \mathrm{~cm}$.

For practical use, Eq. (26) can be simplified as the correlations between expected visibilities are either 0 or 1 when the calibrators are respectively different or alike. The only requirement to compute this correlation is therefore that the variances of the expected visibilities and the coefficients $\alpha$ and $\beta$ be saved with the reduced data. These correlations will have to be computed to model fit the data. The generalization of the Levenberg-Marquardt method with correlated data is given in the Appendix at the end of the paper.

\section{Validation of the method}

Examples of data reduction results and calibrations are presented. The quantities introduced in the previous sections are discussed in practical situations and general comments on observing strategies are expressed.

\subsection{Squared coherence factors statistics}

I have plotted in Fig. 1 three examples of $\mu^{2}$ distributions. In the case of V636 Her, the fringe speed puts the fringe frequency far above the turbulence piston spectrum. The piston is almost frozen during each scan and the amount of correlated noise is small. In the case of $71 \mathrm{UMa}$, the fringe speed is lower and the measurements are more sensitive to piston hence the higher correlated noise. $\delta$ Sge is an intermediate case. In all three examples, the distributions of $\mu^{2}$ are compatible with Gaussian distributions hence validating the basic assumption on the statistics of the $\mu^{2}$. An important fact is that the amount of correlated noise is not negligible and must be taken into account. However, a test on distributions is performed to detect deviations from Gaussian statistics. Deviations are not common and are always due to instrumental problems. In such cases, depending on the required level of data quality, data may be eliminated.

\subsection{Examples of transfer functions}

Figure 2 presents two examples of squared co-transfer functions. Full circles are measurements on calibrators whereas open circles are interpolations for science targets. It is visible that the co-transfer function is not always stable and may experience variations. In some cases like on May 15, 2000 at 8:07, an error of calibration may have happened as the co-transfer functions jump by a few percent. Yet, in most cases, the transfer functions variations are slow on time scales of a few hours and variations can be well approximated to the first order. Data collected on May 22, 2000 show that this is still the case when the calibrator diameters are known with a very good precision.

\subsection{Discussion of model fitting and examples}

\subsubsection{Amount of correlation}

Before presenting examples let us summarize the different levels of correlations we have encountered so far:

1. correlation of coherence factors (coaxial beamcombiners); 
2. correlation of interferometric channels (coaxial beamcombiners);

3. correlation of simultaneous baselines;

4. correlation of non-simultaneous baselines.

The first level $(r)$ was addressed in Sect. 7.1. The amount of correlation between interferometric channels $\left(\rho_{12}\right)$ for a coaxial beamcombiner like FLUOR varies from a few percent for faint sources calibrated by very well-known calibrators to almost $100 \%$ for bright sources calibrated by sources whose diameters are known with an accuracy of a few percent. The two channels are therefore not fully independent in this case and it is important to check the $\chi^{2}$ defined by Eq. (24). A large $\chi^{2}$ may indicate that either the assumptions on Gaussian statistics were wrong for these particular data or that the transfer function variation is not well measured. In either case, data should be examined in detail to decide whether the visibility value can be used or not. A blind method is to reject visibilities with a $\chi^{2}$ above a certain level that can be of 3 for difficult programs or relaxed to a larger value for easier programs. It is important to note that if the transfer function has varied accordingly in both channels at the time the science target was observed by an amount larger than the error bars then this $\chi^{2}$ test will fail to detect it. It can only be detected if the variations are opposite in the two channels. This is certainly a weakness.

It will be interesting to assess the level of correlations of visibilities measured with multiple beam interferometers. It can be anticipated that it will not be negligible and will be of the same level as $\rho_{12}$.

The importance of correlation between visibilities recorded separately is illustrated in Fig. 3. The data have been reduced in two different ways. Data plotted with open circles and fitted by a dashed-line uniform disk model are reduced without taking correlations into account. Data plotted with full circles and fitted by the continuous line were reduced with the method of this paper. In the first case, the fit is of very good quality with a $\chi^{2}$ smaller than 1 . Yet, all visibilities have been calibrated with the same source, hence a strong correlation between visibility values as the $3 \times 3$ correlation matrix shows:

$C=\left[\begin{array}{ccc}1 & 0.96 & 0.96 \\ 0.96 & 1 & 0.97 \\ 0.96 & 0.97 & 1\end{array}\right]$

It is to be noticed that the $\rho_{12}$ correlation factor is larger than $90 \%$ for all three visibilities, a large fraction of this correlation being due to the common calibrator. If correlations are ignored then noise is considered independent from one visibility to the other and this is why the first $\chi^{2}$ is smaller, as a large global noise is now interpreted as a large fluctuating noise from one visibility to the other. On the contrary, when correlations are used, a tiny fraction of noise (4\% at most) can be considered a fluctuation giving degrees of freedom for the adjustement of the model. This is equivalent to reducing error bars on visibilities by $96 \%$ in the fit. The common noise due to the uncertainty on the calibrator is then a simple common bias on the visibilities but does not contribute to the noise in the fit, hence the much larger $\chi^{2}$. In the zoomed part of this same figure, one can see that the fit now conforms to only one of the visibility data as the correlation matrix is close to being non-invertible (see the Appendix for the use of the correlation matrix in the fitting process). In more physical terms, the correlations being very close to one, all data are equivalent and the fit can be derived from one of the visibility data. If all data points were compatible despite the large correlations then the best fit curve would go through the error bars. It is not the case here and this is why the $\chi^{2}$ is large.

\subsubsection{Examples of visibility accuracies}

Other examples of model fitting are presented in Fig. 4. The BK Vir data were calibrated with the same calibrator (the correlation matrix is similar to the matrix above) as SW Vir but the visibilities are very consistent with each others. Data for the three other sources are either totally independent or slightly correlated. Only the first lobe data were used for the fit of $\mathrm{G}$ Her. These four examples show very good fits and consistency of data. In particular, this sets the best absolute accuracy of the calibration of visibilities with FLUOR to 0.004 (equivalent to an accuracy of 0.004 on $V^{2}$ with $V=0.5$ as $\sigma_{V^{2}}=2 \sigma_{V} . V$ to the first order).

\subsubsection{Calibration strategies}

It is important to adapt the strategy of calibration to the type of astrophysical studies addressed with optical interferometers. For most studies where visibility accuracies of a few percent are acceptable, the repeated use of a single or of a few calibrators is possible. For difficult programs like exoplanet detection, a very high level of accuracy is required and the strategy needs to be well prepared. Two cases may arise depending on whether the required calibration of visibilities is absolute or relative. If absolute accuracies better than 0.001 have to be obtained on visibilities then it is very likely that no calibrator can be used twice, unless the error on the expected visibility of this calibrator is less than the level of accuracy required. This would suppose that the visibility model of the calibrator be measured first. Another possibility is relative detection. As illustrated by the example of SW Vir, if the same calibrator is systematically used, the fit is sensitive to very low levels as the correlated noise does not contribute to the value of the $\chi^{2}$. In this example, a departure from the uniform disk model or a calibration error may have been detected to a level much lower than the error bars. For very faint detail detection, this can work if the visibility curve of the calibrator is smooth and without wiggles of similar amplitude as the ones searched for on the science target.

In any case, the observing strategy should be prepared in advance and should take the problem of data correlations into account.

\section{Conclusion}

I have proposed in this paper a method to calibrate visibility data obtained with single mode interferometers. The single mode character is required to make valid the assumption that the statistics of coherence factors data are Gaussian and stationary. It is possible to derive reliable error bars if all correlations are considered in the derivation of all estimators. Correlations 
also need to be taken into account when fitting the data by models. The validity of the method has been demonstrated on real interferometric data recorded with FLUOR. An important conclusion of this work is that the strategy of calibration has to be adapted for specific programs requiring high standards of calibration.

\section{References}

Chelli, A. 2000, AMBER report AMB-IGR-017

Coudé du Foresto, V., Ridgway, S. T., \& Mariotti, J.-M. 1997, A\&AS, 121,379

Mourard, D., Tallon-Bosc, I., Rigal, F., et al. 1994, A\&A, 288, 675
Pelat, D. 1992, Cours Bruits et Signaux, École Doctorale d'Île de France, Astronomie-Astrophysique

Perrin, G. 1996, Ph.D. Thesis, Université Paris VII

Perrin, G., Coudé du Foresto, V., Ridgway, S. T., et al. 1998, A\&A, 331,619

Perrin, G. 2003, A\&A, 398, 385

Press, W. H., Flannery, B. P., Teukolsky, S. A., \& Vetterling, W. T. 1988, Numerical Recipes in C (Cambridge: Cambridge University Press)

Segransan, D., Forveille, T., Millan-Gabet, R., Perrier, C., \& Traub, W. A. 1999, Working on the Fringe: Optical and IR Interferometry from Ground and Space, ed. S. Unwin, \& R. Stachnik, ASP Conf., 194, 290 